\title{
A STUDY OF MONOTONICALLY NORMAL SPACES
}

\author{
CARLOS R. BORGES
}

\begin{abstract}
We obtain various characterizations of monotonically normal spaces which not only answer various questions of Zenor but also allow an elementary proof of a result of Heath and Lutzer. We also prove that elastic spaces are monotonically normal.
\end{abstract}

Recently P. Zenor introduced the class of monotonically normal spaces (his results will appear in [3]). Soon afterward, R. Heath and D. Lutzer proved that each linearly ordered topological space is monotonically normal (see [3]). Since Zenor did not know if monotonically normal spaces were hereditarily monotonically normal and the result of Heath and Lutzer had a very long proof, my interest in the study of these spaces was aroused. The characterizations of monotonically normal spaces which follow not only answer Zenor's questions but also permit us to prove, quite easily, that linearly ordered topological spaces and elastic spaces are monotonically normal. (Our proof of the first result appears in [2]. For the second result, see Theorem 2.3.)

1. Characterizations of monotonically normal spaces. For the sake of completeness, we will first define this class of spaces.

Defínition 1.1. For any space $X$, let $\mathscr{D}_{X}=\{(A, B) \mid A$ and $B$ are disjoint closed subsets of $X$ \}. The $T_{1}$-space $X$ is said to be monotonically normal provided that to each $(A, B) \in \mathscr{D}_{X}$ one can assign an open subset $G(A, B)$ of $X$ such that

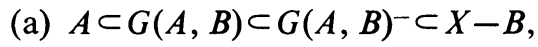

(b) $G(A, B) \subset G\left(A^{\prime}, B^{\prime}\right)$, whenever $A \subset A^{\prime}$ and $B^{\prime} \subset B$.

The function $G$ is called a monotone normality operator.

THEOREM 1.2. The following are equivalent:

(a) $X$ is monotonically normal.

(b) $X$ is $T_{1}$, and to each pair $(A, B)$ of subsets of $X$, with $A \cap B^{-}=\varnothing=$ $A^{-} \cap B$, one can assign an open subset $G(A, B)$ of $X$ such that

(i) $A \subset G(A, B) \subset G(A, B)^{-} \subset X-B$,

(ii) $G(A, B) \subset G\left(A^{\prime}, B^{\prime}\right)$ whenever $A \subset A^{\prime}$ and $B^{\prime} \subset B$.

Received by the editors May 12, 1972 and, in revised form, June 23, 1972.

AMS (MOS) subject classifications (1969). Primary 5420, 5450; Secondary 5456.

Key words and phrases. Monotonically normal, linearly ordered topological spaces, elastic spaces.

(c) American Mathematical Society 1973 
(c) To each pair $(A, U)$ of subsets of $X$, with $A$ closed, $U$ open and $A \subset U$, we can assign an open set $U_{A} \supset A$ such that

(i) $U_{A} \subset V_{B}$ whenever $A \subset B$ and $U \subset V$,

(ii) $U_{A} \cap(X-A)_{X-U}=\varnothing$.

(d) For each open $U \subset X$ and $x \in U$ there exists an open neighborhood $U_{x}$ of $x$ such that $U_{x} \cap V_{y} \neq \varnothing$ implies $x \in V$ or $y \in U$.

(e) There exists a base $\mathscr{B}$ for $X$ such that, for each $B \in \mathscr{B}$ and $x \in B$, there exists an open neighborhood $B_{x}$ of $x$ such that

$$
B_{x} \cap C_{y} \neq \varnothing \text { implies } x \in C \text { or } y \in B .
$$

(f) X satisfies Lemma 2.1 of $[\mathbf{1}]$.

(g) $X$ satisfies Theorem 5.1 of $[1]$.

Proof. The scheme of this proof will be (a) $\Rightarrow(\mathrm{c}) \Rightarrow(\mathrm{d}) \Rightarrow(\mathrm{e}) \Rightarrow(\mathrm{f}) \Rightarrow$ $(\mathrm{g}) \Rightarrow$ (a) and (d) $\Rightarrow$ (b). This suffices since, obviously, (b) implies (a).

(a) implies (c). By [3, Lemma 2.2], $X$ has a monotone normality operator $G$ such that $G(A, B) \cap G(B, A)=\varnothing$ for each $(A, B) \in \mathscr{D}_{X}$ (indeed, the proof consists of observing that, for any monotone normality operator $H$ on $X$, letting $G(A, B)=H(A, B)-H(B, A)^{-}$will do the trick). Now, for each pair $(A, U)$ with $A$ closed, $U$ open and $A \subset U$, let $U_{A}=$ $G(A, X-U)$. It is quite easy to see that conditions (i) and (ii) of (c) are satisfied.

(c) implies (d). For each open $U \subset X$ and $x \in U$, let $U_{x}=U_{\{x\}}$. Suppose $U_{x} \cap V_{y} \neq \varnothing, x \notin V$ and $y \notin U$. Then $U_{x} \cap(X-\{x\})_{X-U}=\varnothing$ and $V_{y} \subset$ $(X-\{x\})_{X-U}$. Consequently $U_{x} \cap V_{y}=\varnothing$, a contradiction. This proves that $U_{x} \cap V_{y} \neq \varnothing$ implies $y \in U$ or $x \in V$.

Clearly (d) implies (e). Therefore we next prove that (e) implies (f). For each pair $(A, U)$ of subsets of $X$, with $A$ closed and $U$ open, let

$$
U_{A}=\bigcup\left\{V_{x} \mid x \in A \text { and } V \subset U\right\} .
$$

First we show that $\left(U_{A}\right)^{-} \subset A \cup U$ : Pick any $x \notin A \cup U$. Then it is easily seen that $(X-A)_{x} \cap U_{A}=\varnothing$. This shows $\left(U_{A}\right)^{-} \subset A \cup U$. Next we show that (iv) is satisfied: Suppose $A \subset U$ and $U_{A} \cap(X-A)_{(X-U)} \neq \varnothing$. Then $V_{a} \cap W_{b} \neq \varnothing$ for some $a \in A$ and $V \subset U, b \in X-U$ and $W \subset X-A$. From (d) we get that $a \in W$ or $b \in V$, a contradiction.

Clearly all other requirements are satisfied.

(f) implies (g). This proof is exactly the same as the proof of Theorem 5.1 of $[1]$.

(g) implies (a). For each pair $(A, B)$ of disjoint closed subsets of $X$, let

$$
G(A, B)=f_{X-B, A}^{-1}\left[0, \frac{1}{2}\right] .
$$

It is easily seen that the conditions (a) and (b) of Definition 1.1 are satisfied. 
Finally we show that (d) $\Rightarrow(\mathrm{b})$. For each pair $(A, B)$ of subsets of $X$ with $A \cap B^{-}=\varnothing=A^{-} \cap B$, let

$$
G(A, B)=\bigcup\left\{U_{x} \mid x \in A, U \subset X-B\right\} .
$$

Clearly we only need to show that $G(A, B)^{-} \subset X-B$. Indeed we prove the stronger result that $G(A, B) \cap G(B, A)=\varnothing$ : Assume there exists $w \in G(A, B) \cap G(B, A)$. Then $w \in U_{a}$ for some $a \in A$ and some open $U \subset X-B$ and $w \in V_{b}$ for some $b \in B$ and some open $V \subset X-A$. Then $U_{a} \cap V_{b} \neq \varnothing$ implies that $b \in U$ or $a \in V$, a contradiction (for example, $b \in U \subset X-B$ contradicts the fact that $b \in B$ ). This completes the proof.

COROLlaRY 1.3. Monotone normality is hereditary.

Proof. Immediate from Theorem 1.2(d).

2. An application. Throughout this section, $E$ is an elastic space (see [5] for definition) and $\mathscr{P}$ is an elastic base for $E$. Furthermore, for $i=1,2$, let $\mathscr{P}_{i}=\left\{P_{i} \mid\left(P_{1}, P_{i}\right) \in \mathscr{P}\right.$ or $\left.\left(P_{i}, P_{2}\right) \in \mathscr{P}\right\}$.

Definition 2.1. For each open $U \subset E$ and $P=\left(P_{1}, P_{2}\right) \in \mathscr{P}$, let

$$
U(P)=\bigcup\left\{V_{1} \mid\left(V_{1}, V_{2}\right) \in \mathscr{P}, V_{2} \subset U \text { and } V_{1} \leqq P_{1}\right\} .
$$

LEMMA 2.2. The sets $U(P)$ satisfy the following conditions:

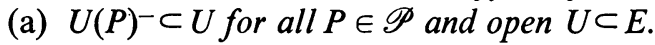

(b) $\bigcup\{U(P) \mid P \in \mathscr{P}\}=U$, for each open $U \subset E$.

(c) $U \subset W, U$ and $W$ open, implies that $U(P) \subset W(P)$ for each $P \in \mathscr{P}$.

(d) If $P_{1} \leqq Q_{1}$ then $U(P) \subset U(Q)$ for all open $U \subset E$.

(e) For each collection $\mathscr{U}$ of open subsets of $E$ and $P \in \mathscr{P}$,

$$
\left(\bigcup\left\{U(Q) \mid Q_{1} \leqq P_{1} \text { and } U \in \mathscr{U}\right\}\right)^{-} \subset \bigcup\{U \mid U \in \mathscr{U}\} .
$$

Proof. Parts (a)-(d) follow easily from the definition of the sets $U(P)$. Part (e) is an immediate consequence of (a), (c) and (d), because

$$
\begin{aligned}
\left(\bigcup \left\{U(Q) \mid Q_{1} \leqq P_{1}\right.\right. & \text { and } U \in \mathscr{U}\})^{-} \\
& \subset[(\bigcup\{U \mid U \in \mathscr{U}\})(P)]^{-} \subset \bigcup\{U \mid U \in \mathscr{U}\} .
\end{aligned}
$$

THEOREM 2.3. Each elastic space E is monotonically normal. (Note that the transitivity of the relation $\leqq$ on $\mathscr{P}_{1}$ is not needed in this result.)

Proof. Because of Theorem 1.2(d), it suffices to show that for each open $U \subset E$ and $x \in U$ there exists an open neighborhood $U_{x}$ of $x$ such that $U_{x} \cap V_{y} \neq \varnothing$ implies $x \in V$ or $y \in U$. This is easily done. Indeed for each open $U \subset E$ and $x \in U$, let $P(U, x)_{1}$ be any element of $\mathscr{P}_{1}$ such that $x \in P(U, x)_{1} \subset P(U, x)_{2} \subset U$. Then let

$$
U_{x}=P(U, x)_{1}-(\overline{X-x)(P(U, x)}) .
$$


Let us now suppose that $U_{x} \cap V_{y} \neq \varnothing, x \notin V$ and $y \notin U$. Then $P(U, x)_{1} \cap$ $P(V, y)_{1} \neq \varnothing$ which implies that $P(U, x)_{1} \leqq P(V, y)_{1}$ or $P(V, y)_{1} \leqq P(U, x)_{1}$. This yields a contradiction. (For example, $P(U, x)_{1} \leqq P(V, y)_{1}$, given that $P(U, x)_{1} \subset P(U, x)_{2} \subset U \subset(X-y)$, implies that $U_{x} \subset P(U, x)_{1} \subset$ $(X-y)(P(V, y))$, in contradiction with $U_{x} \cap V_{y} \neq \varnothing$.) This completes the proof.

Gruenhage [4] has proved that the Sorgenfrey line (the real line with the half-open interval topology) is not elastic, even though it clearly is monotonically normal.

\section{REFERENCES}

1. C. R. Borges, On stratifiable spaces, Pacific J. Math. 17 (1966), 1-16. MR 32 \#6409.

2. - Four generalizations of stratifiable spaces, Proc. Third Prague Sympos. in General Topology (to appear).

3. R. Heath, D. Lutzer and P. Zenor, Monotonically normal spaces, Trans. Amer. Math. Soc. (to appear).

4. G. Gruenhage, The Sorgenfrey line is not elastic, Proc. Amer. Math. Soc. (to appear).

5. H. Tamano and J. E. Vaughan, Paracompactness and elastic spaces, Proc. Amer. Math. Soc. 28 (1971), 299-303. MR 42 \#8446.

Department of Mathematics, University of California, Davis, California 95616 